\title{
Graphology in Psychological Assessment: A Diagnosis in Writing
}

\author{
Pierre E Cronje ${ }^{1}$, Hester E Roets ${ }^{2, *}$ \\ ${ }^{1}$ Clinical Psychologist, Private Practice, Pretoria, SA \\ ${ }^{2}$ University of South Africa, Pretoria, SA \\ *Corresponding Author:Roetshe@unisa.ac.za
}

Copyright (C) 2013 Horizon Research Publishing All rights reserved.

\begin{abstract}
Many clinicians, even those without graphological knowledge, are fascinated by the uniqueness of handwriting.No wonder, since the development of one's handwriting is the result of a unique, complex developmental process.Fascination in handwriting led to the intriguing study of graphology as a means of understanding human behaviour.There are different schools of graphology, one being the 'trait school' which interprets handwriting according to single signs and the other being the holistic approach, which advocates the analysis of handwriting as a Gestalt.The trait-method does not do justice to the multi-faceted nature of personality, but the holistic approach seems to be a powerful expressive projective technique.The reliability of the holistic method was put to the test by comparing clinical findings of a patient to a 'blind' handwriting analysis of the same patient done independently by a professional graphologist.Graphological inferences made from the five (5) unique composite profiles, show strong similarities with diagnoses on Axis I and Axis II $\left(\mathrm{DSM}-\mathrm{IV}^{-T R} \mathrm{TR}^{\mathrm{TM}}\right.$ ) (APA 2000) of the same patients, which confirms the value of graphology in psychological assessment.
\end{abstract}

Keywords Graphology; Handwriting Analysis, Gestalt Approach,Holistic Analysis, Form Standard

\section{Introduction}

'The Moving Finger writes; and, having writ,

Moves on:nor all thy Piety nor wit

Shall lure it back to cancel half a Line,

Nor all thy Tears will wash a Word of it.'

-Omar Khayyam

In the context of handwriting analysis, this poem underpins how powerful and shockingly revealing handwriting can be.Handwriting analysis, as an expressive projective technique, is believed to access the deeper layers of personality structure and perhaps the complexities of personality dynamics and has shown promise as a valuable and reliable measuring instrument for personality assessment (Broschk 2003:14).

Being a clinical psychologist, who in addition, qualified as a professional handwriting analyst, I became fascinated by the potential value of graphology as a procedure in psychological assessment - both in diagnosis and in treatment.In view of the above the following hypothesis was formulated:Should graphology be useful in personality assessment and successfully applied in identifying the state of mind of the writer at the time of writing, it is expected that graphology should also shed some light on clinical syndromes and personality disorders (Axis I and II respectively) of the DSM-IV-TM ${ }^{\mathrm{TR}}$ (2000).

Since there is a direct link between the brain and the psycho-motor movements of writing, underlying psychodynamics play an important role in the eventual subconscious formation of letters. The psychodynamics of handwriting is briefly revisited in the following section.

\section{Psychodynamics of Handwriting}

All action, writing included, starts in the brain.Although the writing instrument is held by the hand (foot/mouth), it is the brain that impels one to write (Hayes 1993:1), thus the writing organ is governed by the central nervous system - a process which is usually unconscious, but most revealing (Nezos 1986:5).

As handwriting is closely tied to impulses from the brain, it is a barometer of moods and of physical, emotional and mental health (Branston 1998:2; Grandin 1994a:1; Hayes 1993:2).Not only is it a physiological muscular activity - it is a unique personal symbol and an expression of the whole personality (Mendel 1982:17).

The special link between graphological features and personality traits is illustrated in the model in Annexure A.In collaboration with Grandin (2008) I adjusted and elaborated the well-known Roman model $(1952: 131)$ to include a greater variety of intra- and interpsychic processes.

\section{Status of Research in Graphology}


Before I undertook the empirical study to assess the viability of graphology in psycho-educational assessment and psychotherapy, I reviewed the existing research since 1975 regarding the use of graphology. The findings regarding the use of graphology in assessment range from rather negative to highly favorable.

My first observation was that the literature focused mainly on four (4) research areas, namely the use of graphology in:

- assessing aspects of personality and emotional adjustment

- predicting academic/work performance

- identifying leadership potential

- recruitment and personnel selection (Cronje 2009:5)

My second observation was that research in graphology was most popular in the area of recruitment and personnel selection; based on the fact that the majority of articles had recruitment and personnel selection as an objective.My third observation was that the method of analyses most frequently used, was that of the 'trait-method' and very little attention was given to the holistic, Gestalt approach (Cronje 2009:11).

Furthermore, 'poor research designs' (Wallner 1975:8) and 'significant methodological negligence' (Klimonski\&Rafaeli 1983:191), have often led to the unfair rejection of graphology as a unitary phenomenon, rather than to criticize the specific school of research, for example the 'trait school'. Greasley (2000:45) confirmed that negative research findings in graphological personality assessment are due to unclear interpretative procedures and an over-emphasis on the importance of 'fixed signs' ('trait school') rather than a more holistic, intuitive approach.

In favour of the holistic approach to an analysis, the researchers admitted that 'linear relations between individual isolated graphometric indicators and personality/behaviour are unrealistic, since graphology does not operate in a linear pattern' (Nevos 1989:1331).In fact, many a researcher conceded afterwards that a holistic approach is the only accountable method of handwriting analysis in personality assessment.Ironically enough, this viewpoint has been held for decades by pioneers in the field of graphology (Jacoby 1991:42; Mendel 1982: 287; Roman 1952:132) and emphasized by Grandin (2006a:3); Hayes (1993:20) and Nezos (1986:207).

In view of the above, I was convinced that a holistic approach was the only viable and accountable option in research in graphology.The specific methodology of a holistic analytical approach is discussed in more detail in the following paragraph/section.

\section{Methodology of a Holistic Approach}

Very few text books provide the methodology of a holistic approach.Instead, text books usually focus on the different graphological features, their numerous subcategories and possible psychological interpretations of each of these features.A novice in graphology can easily fall in the trap of the mere identifying and matching of traits to characteristics ('trait-method') and would eventually lead to a piecemeal approach to interpretation and negating the unique composition of personality and behaviour at the same time.

The holistic approach on the other hand requires a specific methodology for proper assessment of the handwriting to avoid haphazard conclusions and deductions.According to this interpretative approach, each outstanding graphological feature should be seen in conjunction with other confirmatory features, since certain graphological features tend to convey the same tendency whereas others may contradict.Seemingly contradictory graphological features may, however, co-exist in a writing sample.

This potentially conflicting data may cause disharmony within the individual or they may co-exist in complementary harmony.

By systematically studying all the graphological features indices in the writing, the graphologist can arrive at an overall picture of the writer's personality (Branston 1998:111; Gullan-Whur 1998:28-29 \&Lazewnik 1990:8-9).

Holistic graphology is both an art and a science.Steps in the holistic approach are as follows and each one will be discussed briefly:

- Assessing the Gestalt (over-all view) of the writing

- Assessing the form standard of the writing; be it low or high

- Identifying dominant -, subdominant - andcounterdominant features in the writing

- Interpreting the co-existing features and compiling the synthesis (Cronje 2009:66)

Firstly, the gestalt (overall impression of the handwriting) should be assessed in terms of harmony, consistency, spontaneity and dynamics to mention a few (Grandin 2006a:4-5).This initial, sensory impression of the writing is based on the examiner's intuition and depicts the art of graphology.

The gestalt assessment is followed by assessment of the form standard of the writing (Barrett 1995:12; Branston 1998:110) which is the first step in the scientific assessment of the writing. One such a system is the comprehensive one by Mendel (Mendel 1982: 50-53). The assessment for form standard consists of objective criteria for each of the following aspects: rhythm, symmetry, creativity, speed and legibility, whereupon a numeric value is allocated.

The form standard, be it low or high, is of utmost importance since it directs the tone of interpretation of the features, either predominantly positive or negative.For example, large writing in a handwriting sample with good form standard may mean confidence, self-expression, energy and activity. Should the form standard be low, a large writing may mean arrogance, brusqueness and impulsiveness (Hayes 1993:20).Annexure B shows the differences between two large handwritings, one with a high form standard and the other one with a low form standard.

Assessment of the form standard is followed by identifying the dominant/striking/prominent features, followed by subdominant features (accidental or isolated traits) and the presence of counter-dominant 
features.Counter-dominants are those features that co-exist in a writing which may have contradictory tendencies (Grandin 1994c:110; Gullan-Whar 1998:22 \& Roman 1952:119).

The final step is synthesizing the results by constructing the separate psychological components into an integrated, cohesive whole (Grandin 1994c:110; Hayes 1993:101 \&Lazewnik 1990:8). Such a cohesive synthesis is tailor-made to each individual and provides valuable information about personality factors, interaction style, thinking style as well as subconscious drives which are of diagnostic value to the clinician.Furthermore, such a synthesis can provide useful guidelines for psychotherapy based on hypotheses formulated on the basis of the composite report.

To determine the viability of graphology in psychodiagnosis and -treatment, each of the writings used in empirical study, was assessed by the independent graphologist according to the holistic procedure as outlined above.

\section{Design of the Present Study}

\section{- Sample}

From approximately 1000 samples of handwriting gathered in my clinical practice, a selection of five (5) handwriting samples was made to illustrate the viability of graphology in clinical practice for both diagnosis and treatment.

\section{- Qualitative approach in research}

As a handwriting sample is not quantifiable, a quantitative approach to research in handwriting is not considered a suitable option. The purpose of the present study was indeed to infer and derive diagnostic - and treatment guidelines on the basis of the individualized graphological assessments. The choice of research was qualitative, as the aim of such an approach is to explain data, to confirm, to modify or to elaborate on existing findings.

\section{- Procedure}

The five (5) samples were analyzed in a structured way, according to the recommended holistic approach described earlier.Comments were given on the client's way of thinking, intellectual level, aspirations, social and emotional adjustment, interpersonal style, outstanding characteristics as well as subconscious drives.

To enhance objectivity and validity of findings, an independent, professional graphologist was involved to do the assessments. The principle of a 'blind analysis' done by an independent graphologist has already been recommended by Wallner (1975:14).Nevos (1989:1331) confirmed that graphological analyses should be executed 'blindly' in order to enhance validity and objectivity.The only information given to the independent examiner was the gender of the writer, the age of the writer and whether the writer was either a left-hander or a right-hander.

Findings in the handwriting were compared to my clinical assessment of the patient to cross-validate
findings.Guidelines for the appropriate treatment of each of the participants were formulated according to clinical findings as well as findings form the graphological analyses.

\section{Findings of the Study}

The present study confirmed the positive correlation between the holistic graphological findings and clinical and personality assessment (Axis I and Axis II) on the DSM-IV-TR ${ }^{\mathrm{TM}}$, the two designated axes for intra- and interpsychic processes.Graphology can thus be regarded a useful tool and viable option in psychological assessment and diagnosis.

Although a graphologist does not necessarily use psychological jargon in the reports, as psychology may not also be a field of expertise of the graphologist, the extensive derivations made form a single handwriting sample provided important guidelines in 'picturing' the client/patient.

The reports, furthermore, illustrate that handwriting analysis can access the deeper and complex layers of personality structure and dynamics, even the information the client/patient does not necessarily disclose during the initial therapy sessions due to resistance, shame or guilt.

The graphological assessments do not only correspond with the clinical findings, they also provide reassuring confirmation of clinical findings and provide meaningful inputs for constructing a treatment plan for psychotherapy.

The findings of the current research confirmed the usefulness of graphology in psychological assessment with special reference to the following advantages:

- graphology can help psychologists to understand unconscious mechanisms and the individual feelings and perceptions of their patients

- handwriting analysis reveals the patient's strengths and weaknesses

- the presence of past trauma can be detected by graphology or it may path the way of a discussion on parental influences on the development of the character

- a graphological analysis can provide an extremely useful guideline when establishing therapeutic goals and can pinpoint where treatment is needed

- the usefulness of graphology lies in its ability to fill gaps in knowledge which other sources of information are not able to reveal

- graphology provides confirmation of results obtained from other forms of assessment

- graphology furnishes the psychologist with a blend of clinical, dynamic and unconscious factors in order to obtain a comprehensive picture or the client

\section{Conclusion}

Although the holistic approach requires initial intensive study and experience to master the complex procedure of 
observation, analysis and synthesis, it is definitely a worthwhile endeavor for any clinician.A clinician equipped with a thorough knowledge of graphology, will definitely have an advantage to those who do not have this knowledge.

This valuable assessment procedure is easily administered, culture fair, cannot become obsolete and does not require expensive test material.

Graphology, specifically the holistic approach to analysis, is most definitely a viable option in psychological and clinical assessment.

A handwriting sample, as unique as a fingerprint, is easily obtainable.A person's handwriting seems to be rather consistent over time, despite intra-individual variation and shows potential as a culture fair assessment tool.It does not claim to be infallible, but promise to be a useful and viable tool for the psychologist in understanding the human psyche.

\section{Appendix A:}

\section{Graphological features and Personality}

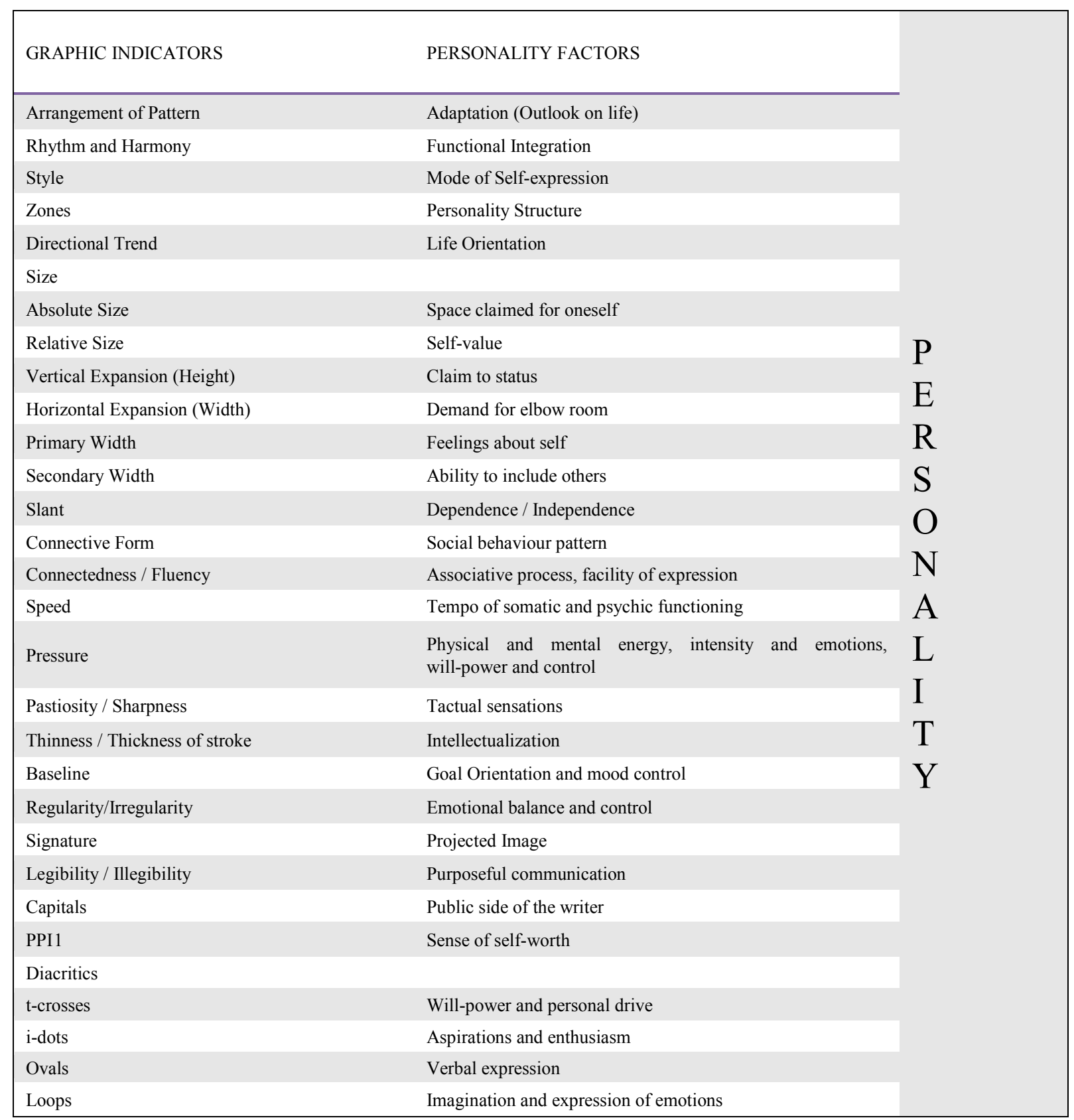

1 Personal Pronoun "I" (PPI) 
Universal Journal of Psychology 1(4): 163-168, 2013

167

Appendix B:

1. An example of large writing with good form standard

Have finished with clients.

off to the 100, if you come past t door is closed.

will stick around until 12 hoo.

Then have to pick up christopher four play school.

The form standard is high, since the writing is symmetrical, creative and rhythmic.

2.An example of large writing with low form standard

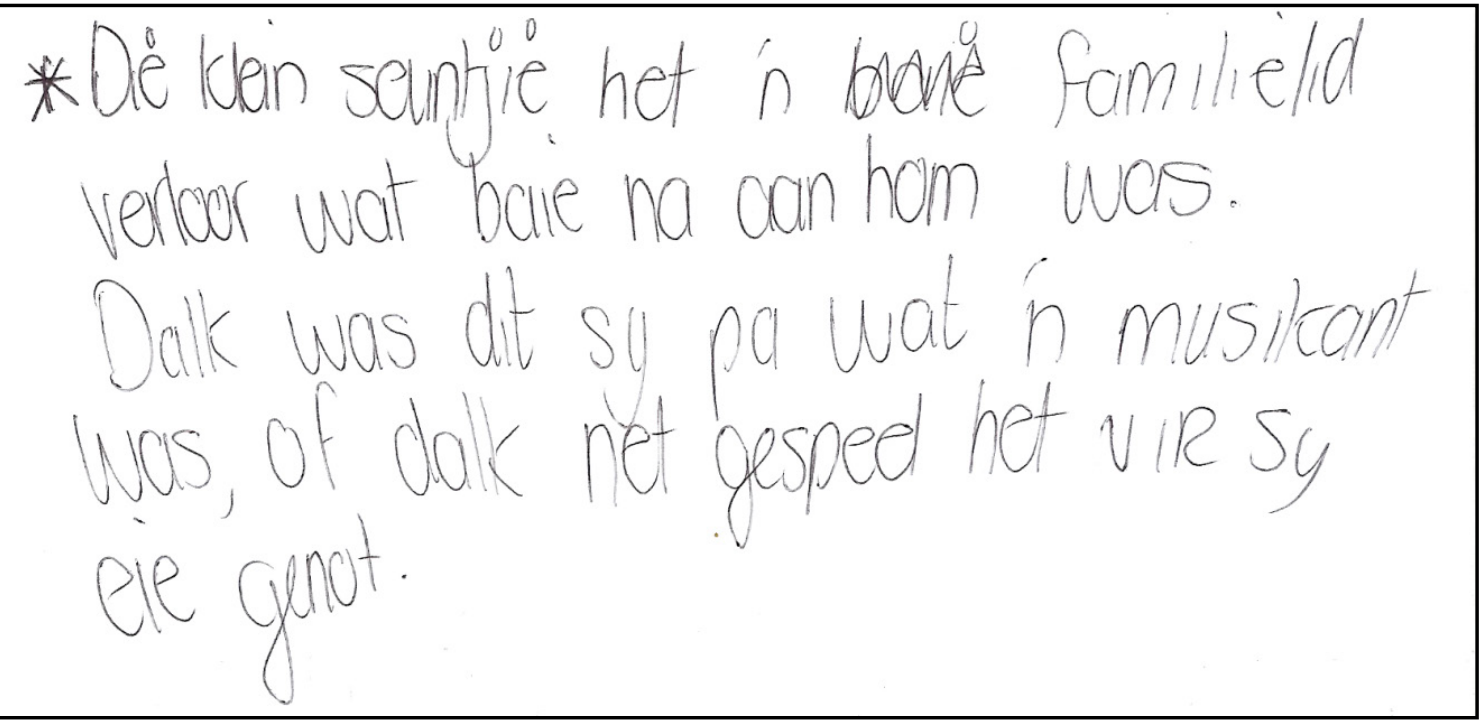

The form standard is low, because it is asymmetrical, arrhythmic, dull and boring. 


\section{REFERENCES}

[1] American Psychiatric Association (APA). DSM-IV-TRTM APA: Washington DC, 2000.

[2] Barrett, D.V. Graphology. The predictions library. London: Dorling Kindersley, 1995.

[3] Branston, B. The elements of graphology. Shaftesbury: Element Books Limited, 1998.

[4] Broschk, S. Graphology and Personality: A correlational analysis. Unpublished Master's dissertation, Johannesburg: Rand Afrikaans University, 2003.

[5] Cronje, P.E. The viability of graphology in psycho-educational assessment. Unpublished D Ed thesis, Pretoria: University of South Africa, 2009.

[6] Grandin, S. Gaining insight into human nature through graphology. Textbook for the Beginner/ Intermediate course of Handwriting analysis. Johannesburg: Grafex, 1994a.

[7] Grandin, S. Gaining insight into human nature through graphology: Textbook for the advanced course. Johannesburg: Grafex, 1994c.

[8] Grandin, S. Gaining insight into human nature through graphology. Textbook for the Beginner/ Intermediate course of Handwriting analysis. (Part I) Johannesburg: Grafex, $2006 a$.

[9] Grandin, S. Elaboration on Roman's Model of personality, personal communication, August 23, 2008.

[10] Greasley, P. Handwriting analysis and personality assessment: The creative use of analogy, symbolism and metaphor,

European Psychologist, 5(1): 44-51, 2000. .

[11] Gullan-Whur, M. The secrets of your handwriting (3rd ed.). London: The Aquarian Press, 1998.

[12] Hayes, R. Between the lines. Understanding yourself and others through handwriting analysis. Vermont: Destiny Books, 1993.

[13] Jacoby, H.J. Analysis of Handwriting. An introduction into Scientific Graphology. London: George Allen \&Unwin Ltd, 1991.

[14] Klimoski, R.J. \&Rafaeli, A. Inferring personal qualities through handwriting analysis. Journal of Occupational Psychology, 56(30): 191-202, 1983.

[15] Lazewnik, B.M. Handwriting analysis. A guide to understanding personalities. Pennsylvania: Whitford Press, 1990.

[16] Mendel, A.O. Personality in Handwriting. A handbook of American graphology. New York: Stephen Daye Press, 1982.

[17] Nevos, B. Validation of graphology through use of a matching method based on ranking. Perceptual and Motor Skills, 69 (3, Pt II): 1331-1336, 1989.

[18] Nezos, R. Graphology. The interpretation of Handwriting. London. Rider \& Company, 1986.

[19] Pogorelsky, G. Using graphology in recruitment. People Dynamics, 14 (9): 24-28, 1996.

[20] Roman, K.G. Handwriting. A key to personality. New York: Pantheon Books, 1952.

[21] Rosa, C. Recruitment: the effectiveness of the job applicant selection process. Management Today, 24 (3): 61-63, 2008.

[22] Wallner, T. Hypotheses of handwriting psychology and their verification. Professional Psychology, 6 (1): 8-16, 1975. 